TITLE:

\title{
Electronic excitation and ionization spectra of cyclopentadiene: Revisit by the symmetry-adapted cluster- configuration interaction method
}

\section{$\operatorname{AUTHOR}(S)$ :}

Wan, J; Ehara, M; Hada, M; Nakatsuji, H

\section{CITATION:}

Wan, J ... [et al]. Electronic excitation and ionization spectra of cyclopentadiene: Revisit by the symmetry-adapted cluster-configuration interaction method. JOURNAL OF CHEMICAL PHYSICS 2000, 113(13): 5245-5252

ISSUE DATE:

2000-10-01

URL:

http://hdl.handle.net/2433/50179

\section{RIGHT:}

Copyright 2000 American Institute of Physics. This article may be downloaded for personal use only. Any other use requires prior permission of the author and the American Institute of Physics. 


\title{
Electronic excitation and ionization spectra of cyclopentadiene: Revisit by the symmetry-adapted cluster-configuration interaction method
}

\author{
Jian Wan, Masahiro Ehara, Masahiko Hada, and Hiroshi Nakatsuji ${ }^{a}$ \\ Department of Synthetic Chemistry and Biological Chemistry, Graduate School of Engineering, \\ Kyoto University, Sakyo-ku, Kyoto 606-8501, Japan
}

(Received 26 May 2000; accepted 7 July 2000)

\begin{abstract}
Electronic excitation and ionization spectra of cyclopentadiene $(\mathrm{CP})$ were reinvestigated by the symmetry-adapted cluster (SAC) and SAC-configuration interaction (SAC-CI) method with an extended basis set and a wide active orbital space. To give a satisfactory interpretation of the general profile of the observed excitation spectrum, 40 low-lying excited singlet and triplet states (with excitation energies of up to $9.5 \mathrm{eV}$ ) were computed. The calculated excitation energies were greatly improved compared to those reported previously. All of the peaks in the experimental spectrum were reassigned theoretically with small deviations. The natures of the low-lying valence and Rydberg-excited states were discussed in detail, and the results were also compared with those of some other recent theoretical studies. The ionization energies calculated by the SAC-CI general-R method agree well with the experimental peaks in the photoelectron spectrum. A number of two-electron shake-up states were calculated below $23 \mathrm{eV}$. (C) 2000 American Institute of Physics.
\end{abstract} [S0021-9606(00)30437-8]

\section{INTRODUCTION}

Electronic excited states of cyclopentadiene $(\mathrm{CP})$ have been extensively investigated by various spectroscopic experiments due to its importance as a model compound for photochemical reactions of various cyclic dienyl systems. The locations and assignments of various singlet intravalence $\pi-\pi^{*}$ and Rydberg transitions have been a major subject of optical ultraviolet (UV) spectroscopic studies. ${ }^{1-3}$ Spinforbidden singlet-triplet transitions have so far been investigated by electron energy-loss (EEL) spectroscopy. ${ }^{4}$ Ionization potentials (IPs) of $\mathrm{CP}$ have also been studied by photoelectron spectroscopy (PES). ${ }^{5}$

On the other hand, the experimental work has received relatively little support from $a b$ initio calculations. Only a few theoretical interpretations of observed spectra based on $a b$ initio calculations have been reported; symmetry-adapted cluster (SAC) and SAC-configuration interaction (SAC-CI) calculations ${ }^{6}$ in 1985, random phase approximation (RPA) calculations $^{7}$ in 1991, complete active space second-order perturbation (CASPT2) calculations ${ }^{8}$ in 1993, and multireference Møller-Plesset (MRMP) perturbation calculations ${ }^{9}$ in 1996. The earliest SAC/SAC-CI study ${ }^{6}$ was a pioneering work, but because of the lack of the computational resources in those days, the limited basis sets and small active space impaired the quality of the results. The RPA calculations were not accurate enough to allow a full interpretation of the spectrum. In the latter CASPT2 and MRMP studies, fairly large basis sets were used; an atomic natural orbital (ANO) basis set augmented by ring-centered diffuse $s-, p$ - and $d$-functions in CASPT2, and Dunning's cc-pVTZ basis set augmented by charge-centered diffuse $s-, p$-, and $d$-functions

\footnotetext{
a) Author to whom correspondence should be addressed. Electronic mail: hiroshi@sbchem.kyoto-u.ac.jp
}

in MRMP. These two similar theoretical methods gave very similar computational results and gave a relatively reasonable interpretation of singlet valence excitations lower than 8 $\mathrm{eV}$. The ionized states of $\mathrm{CP}$ were investigated by, together with the excited states, the SAC/SAC-CI method, ${ }^{6}$ but not by the CASPT2 and MRMP methods.

In this study, we re-examined the singlet and triplet valence and Rydberg excitations and ionizations of CP using a better-quality basis set and a larger active space by the SAC/ SAC-CI singles and doubles operator $R_{K}^{+}$(SD-R) calculations. Furthermore, to give a theoretical interpretation of correlation (satellite) peaks characterized by the multielectron process in the PES spectrum, ${ }^{5}$ the SAC/SAC-CI general-R method $^{10}$ was also employed for an accurate calculation of the inner-valence region of the ionization spectrum. In Sec. II, we outline the computational details. Calculated results and discussions are presented in Sec. III. A summary is given in Sec. IV.

\section{COMPUTATIONAL DETAILS}

The details of the SAC/SAC-CI method for calculating ground, excited, and ionized states of molecules have been presented elsewhere. ${ }^{10-18}$ An experimental ground-state equilibrium geometry of CP with $C_{2 v}$ symmetry ${ }^{19}$ was used and the $\mathrm{CP}$ ring was put on the $y z$-plane with $z$ as the principle axis (Fig. 1). The calculated excitation energies are, therefore, vertical in nature.

Dunning's augmented correlation consistent basis set AUG-cc-pVDZ (Ref. 20) was used for C atoms, and ccpVDZ (Ref. 21) was used for $\mathrm{H}$ atoms. Additionally, a set of diffuse functions $(3 s 3 p 3 d)$ selected from the studies of Kaufmann et al. ${ }^{22}$ was placed on the molecular center of gravity. These molecule-centered diffuse functions are the same as those used by Trofimov and Schirmer. ${ }^{23}$ All SCF 


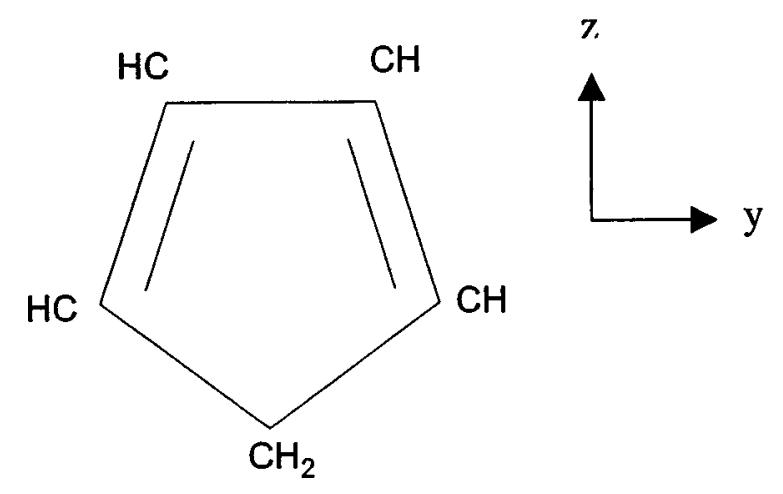

FIG. 1. Cyclopentadiene $\left(\mathrm{C}_{5} \mathrm{H}_{6}\right)$ molecule.

calculations were performed with the GAUSSIAN 98 package. ${ }^{24}$ The total number of basis functions is 185 . This augmented basis set is adequate for describing widely different electronic structures of molecules in the ground state, singlet and triplet valence and Rydberg excited states and ionized states, and for describing valence and diffuse characters and different correlation effects.

The present SAC/SAC-CI calculations were performed with the local version of the SAC-CI module. ${ }^{18}$ The active space consists of 13 occupied orbitals and 167 virtual orbitals; only the $1 s$ core MOs of $\mathrm{C}$ atoms were frozen. In the SAC ground-state calculation, all single-excitation and selected double-excitation operators $S_{I}^{+}$were included in the linked term. The energy threshold $\lambda_{g}$ for perturbation selection $^{16}$ (PS) was $1.0 \times 10^{-5}$ a.u. For the unlinked term, we included only the products of the double-excitation operators $S_{I}^{+} S_{J}^{+}$when the coefficients $C_{I}$ and $C_{J}$, estimated by SD-CI in practice, were larger than $1.0 \times 10^{-3}$.

In the SAC-CI SD-R calculations of excited and ionized states, all single-excitation operators and selected doubleexcitation operators were included in the linked operators $R_{K}^{+}$. Perturbation selection was performed as follows. First, we selected the main reference configurations from SE-CI with a coefficient greater than 0.1 , and then selected the double-excitation operator whose second-order perturbation matrix element with one of the main reference configurations is larger than the threshold $\lambda_{e}\left(1.0 \times 10^{-6}\right.$ a.u. $)$. In the unlinked term $\Sigma_{K, I} d_{K} C_{I} R_{K}^{+} S_{I}^{+}|0\rangle$, we included the doubleexcitation operators $\left\{S_{I}^{+}\right\}$whose coefficients $\left(C_{I}\right)$ were larger than $1.0 \times 10^{-3}$ in the SAC ground-state calculation, and as the $R_{K}^{+}$operator, we included the single- and doubleexcitation operators whose coefficients (taken from SD-CI in practice) were greater than 0.05 .

In the SAC-CI general-R (Ref. 10) calculations of the outer- and inner-valence ionization potentials of $\mathrm{CP}$, a preliminary SD-CI calculation was performed to select the operators for the exponential generation (EG) scheme $^{17}$ by which we construct important triple and quadruple excitation operators required for a quantitative description of the multielectron process in the ionization spectrum. The generated higher-order excitation operators were further selected by the perturbation selection ${ }^{16}$ procedure with the energy threshold of $1.0 \times 10^{-6}$ hartree. Therefore, all single (ionization) operators and selected higher operators (SDTQ) were included in the linked term of SAC-CI general-R equation. In the unlinked term, we included all the double-excitation operators $\left\{S_{I}^{+}\right\}$in the SAC ground-state calculation, and as the $R_{K}^{+}$ operator, we included all single operators and selected higher-order operators up to quadruple.

Table I shows the previous ${ }^{6}$ and present SAC/SAC-CI SD-R computational conditions. Fifteen years ago, the computational resources were substantially smaller than those used here; the available main memory and disk space were very limited, though the previous calculations were certainly "big" by the standard of that time. With the tremendous advances in computer technology, we can use a much better basis set and much larger active space. This improvement, by itself, made the calculated results significantly better, even

TABLE I. Comparison of the previous and present SAC/SAC-CI computational conditions for cyclopentadiene.

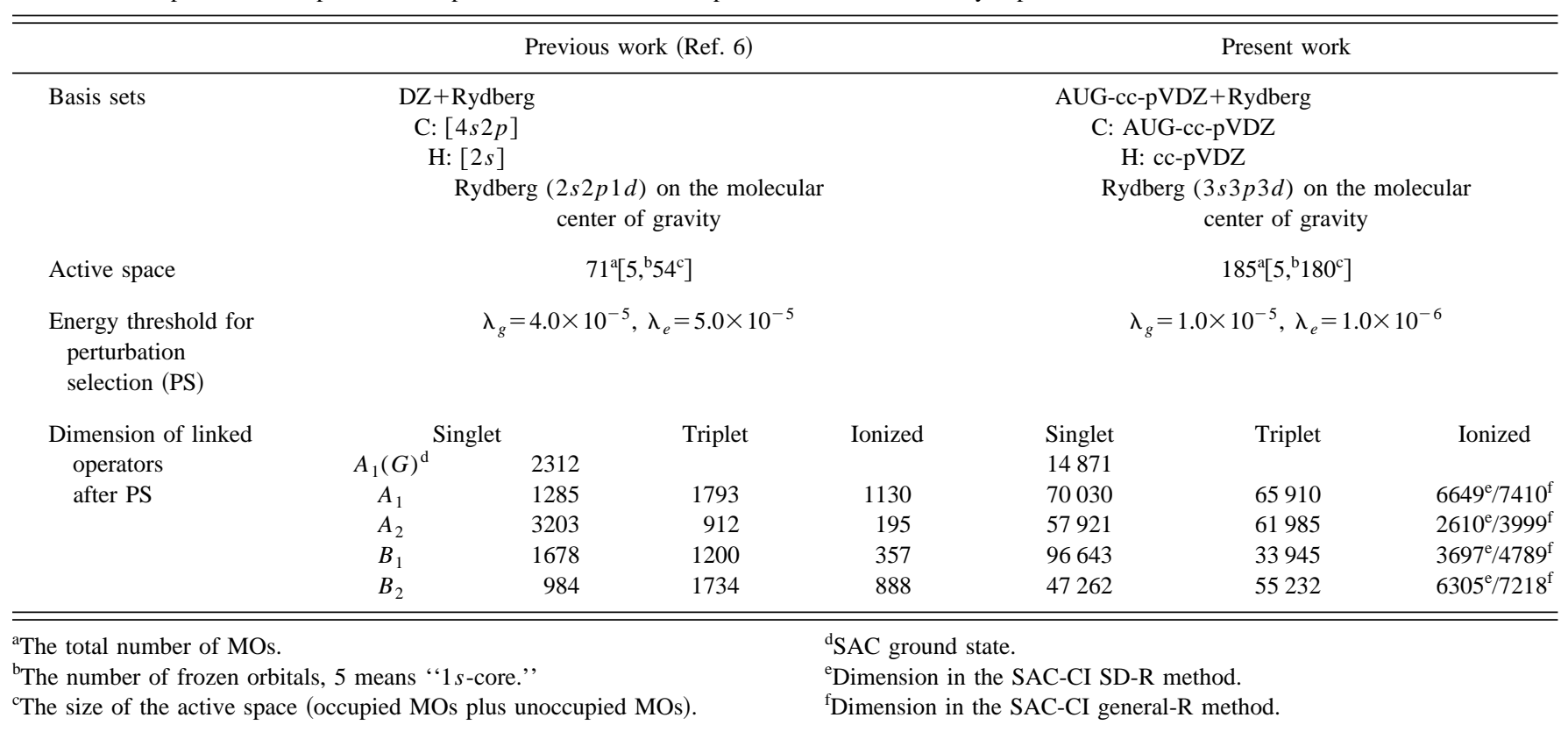


though the basic method used here is the same as the previous one, i.e., the SAC/SAC-CI method. The improvements in the SAC/SAC-CI program and algorithm, etc., are also important, but for the comparisons in Table I, the improvements in the computer technology play the greatest role.

\section{RESULTS AND DISCUSSION}

With the basis set mentioned above, the SCF energy of $\mathrm{CP}$ is -192.814698 hartrees. The SAC ground-state energy is -193.172258 hartrees, and the correlation energy is 0.357559 hartrees. The corresponding previous values were $-192.73732,-192.96584$, and 0.22852 hartrees, respectively.

To distinguish between valence and Rydberg-type transitions, the second moments of the charge distribution $\left\langle r^{2}\right\rangle$ and its components $\left\langle x^{2}\right\rangle,\left\langle y^{2}\right\rangle$, and $\left\langle z^{2}\right\rangle$ were calculated. Since all of the $\pi-\pi^{*}$ transitions in CP belong either to $A_{1}$ or $B_{2}$ symmetry, the $A_{2}$ and $B_{1}$ Rydberg states are not perturbed by the valence $\pi-\pi^{*}$ transitions. The oscillator strength has also been used by several authors as an indicator of valence versus Rydberg character in the case of strong mixing of valence and Rydberg states. We will use all these knowledge to analyze the characters of the excited states.

The SCF occupied valence orbitals and some important virtual orbitals are shown in Table II, together with their orbital energies, symmetries, and characters. Table III shows singlet and triplet excitation energies, oscillator strengths and second moments calculated by the SAC/SAC-CI SD-R method. In Table IV, the singlet and triplet excitation energies calculated by the SAC-CI SD-R method are compared to the experimental values, and to the CASPT2 and MRMP results. Figure 2 shows a comparison of the experimental EEL spectrum ${ }^{4}$ and the theoretical SAC-CI spectrum of CP.

\section{A. Singlet valence excited states}

Roughly speaking, CP is regarded as two ethylene $\pi$ systems with two occupied $\pi$ and two antibonding $\pi^{*}$ molecular orbitals (MO's). Therefore, there are four separate singlet excited states within these $\pi$ MO's, and Mulliken ${ }^{25}$ designated the four lowest $\pi-\pi^{*}$ singlet transitions in this simple model as $N \rightarrow V_{1}, V_{2}, V_{3}$, and $V_{4}$ in the order of the transition energy. All of these excitations are electric-dipole allowed, and the transitions $N \rightarrow V_{1}, V_{2}, V_{3}$, and $V_{4}$ are characterized as ${ }^{1} B_{2},{ }^{1} A_{1},{ }^{1} A_{1}$, and ${ }^{1} B_{2}$, respectively, due to their irreducible representations.

The lowest observed ${ }^{3}$ singlet transition, $N \rightarrow V_{1}\left(\widetilde{X}^{1} A_{1}\right.$ $\rightarrow{ }^{1} B_{2}$ ) designated as peak I in Fig. 2, begins at $4.75 \mathrm{eV}$ and ends at $5.90 \mathrm{eV}$ with the absorption maximum located at $5.34 \mathrm{eV}$. Frueholz et al. ${ }^{4}$ observed the intensity maximum of a broad transition at $5.26 \mathrm{eV}$ by electron impact spectroscopy and assigned it to $N \rightarrow V_{1}\left(\widetilde{X}^{1} A_{1} \rightarrow{ }^{1} B_{2}\right)$. Our current lowest singlet excited state was computed to be $1{ }^{1} B_{2}$ at $5.54 \mathrm{eV}$ with oscillator strength of 0.1305 , which is clearly related to the intense feature of peak I. The second moment $\left\langle r^{2}\right\rangle$ is 72.35 a.u. $^{2}$, which clearly means a valence-excited state. The nature of the $1{ }^{1} B_{2}$ state is $\pi-\pi^{*}$ excitations from the HOMO ( $1 a_{2}: 18$ th MO) to the virtual $\pi^{*}$ orbital (48th MO). There is very little mixing of Rydberg functions.
TABLE II. Hartree-Fock occupied valence molecular orbitals (MOs) and important unoccupied MOs.

\begin{tabular}{|c|c|c|c|}
\hline MOs & Symmetry & Orbital energy (eV) & Nature \\
\hline \multicolumn{4}{|l|}{ Occupied: } \\
\hline Cores & & & $\sigma(1 s)$ \\
\hline 6 & $4 a_{1}$ & -31.362 & $\sigma(2 s)$ \\
\hline 7 & $3 b_{2}$ & -26.302 & $\sigma(2 s)$ \\
\hline 8 & $5 a_{1}$ & -26.000 & $\sigma(2 s)$ \\
\hline 9 & $6 a_{1}$ & -20.571 & $\sigma(2 s)$ \\
\hline 10 & $4 b_{2}$ & -19.811 & $\sigma(2 s)$ \\
\hline 11 & $7 a_{1}$ & -18.944 & $\sigma(2 p)$ \\
\hline 12 & $1 b_{1}$ & -16.071 & $\pi$ \\
\hline 13 & $5 b_{2}$ & -15.290 & $\sigma(2 p)$ \\
\hline 14 & $8 a_{1}$ & -14.386 & $\sigma(2 p)$ \\
\hline 15 & $9 a_{1}$ & -13.786 & $\sigma(2 p)$ \\
\hline 16 & $6 b_{2}$ & -13.617 & $\sigma(2 p)$ \\
\hline 17 (next HOMO) & $2 b_{1}$ & -11.244 & $\pi$ \\
\hline 18 (НОМО) & $1 a_{2}$ & -8.368 & $\pi$ \\
\hline \multicolumn{4}{|l|}{ Unoccupied } \\
\hline 19 (LUMO) & $a_{1}$ & 0.048 & $s$-Rydberg \\
\hline 25 & $b_{2}$ & 0.345 & $d_{y z}$-Rydberg \\
\hline 29 & $a_{1}$ & 0.494 & $s$-Rydberg \\
\hline 31 & $b_{2}$ & 0.561 & $p_{y}$-Rydberg \\
\hline 33 & $a_{1}$ & 0.955 & $s / d_{y z}$-Rydberg \\
\hline 34 & $b_{2}$ & 0.958 & $d_{y z}$-Rydberg \\
\hline 48 & $b_{1}$ & 2.938 & $\pi^{*}$ \\
\hline 51 & $b_{2}$ & 3.696 & $\sigma^{*}$ \\
\hline 54 & $a_{2}$ & 4.095 & $\pi^{*}$ \\
\hline 56 & $b_{2}$ & 4.585 & $\sigma^{*}$ \\
\hline 58 & $b_{2}$ & 4.861 & $\sigma^{*}$ \\
\hline 59 & $a_{1}$ & 4.883 & $\sigma^{*}$ \\
\hline 63 & $b_{1}$ & 6.069 & $\pi^{*}$ \\
\hline 66 & $b_{2}$ & 7.344 & $\sigma^{*}$ \\
\hline 67 & $a_{2}$ & 7.362 & $\pi^{*}$ \\
\hline
\end{tabular}

At $6.15-6.7 \mathrm{eV}$, there is another feature, peak II, as shown in Fig. 2. This region is characterized by Rydberg transitions, and therefore will be discussed in the next section.

The region with the most intense inelastic scattering in the electron impact spectrum occurs from approximately $7.2-8.7 \mathrm{eV}$, with the intensity maximum located at $7.88 \mathrm{eV}$ (peak III in Fig. 2). Robin ${ }^{26}$ suggested that the corresponding feature $(8.06 \mathrm{eV})$ in the optical spectrum results from the superposition of the $N \rightarrow V_{2}\left(\widetilde{X}^{1} A_{1} \rightarrow{ }^{1} A_{1}\right)$ and $N$ $\rightarrow V_{3}\left(\widetilde{X}^{1} A_{1} \rightarrow{ }^{1} A_{1}\right)$ excitations. Our present calculated results give the $6{ }^{1} B_{2}, 5{ }^{1} A_{1}, 7{ }^{1} B_{2}, 6{ }^{1} A_{1}$, and $7{ }^{1} A_{1}$ states in this region. Although there is extensive mixing of Rydberg and valence transitions in the $6{ }^{1} B_{2}, 7{ }^{1} B_{2}, 6{ }^{1} A_{1}$, and $7{ }^{1} A_{1}$ states, we assign these states to Rydberg transitions according to their larger second moments, weaker oscillator strengths, and existence of Rydberg-type main configurations. The $5{ }^{1} A_{1}$ state is calculated at $8.17 \mathrm{eV}$ with an oscillator strength of 0.5377 , which is the strongest transition in the entire energy region of interest. The second moment $\left\langle r^{2}\right\rangle$ is 152.02 a.u. ${ }^{2}$, which clearly shows a diffuse character (the $\left\langle r^{2}\right\rangle$ of the ground state is 68.46 a.u. $\left.{ }^{2}\right)$, and there is strong Rydberg-mixing in this valence-excited state. The $5{ }^{1} A_{1}$ state is described by the mixed $\pi-\pi^{*}$ excitations from HOMO $\left(1 a_{2}: 18\right.$ th MO) and next HOMO $\left(2 b_{1}: 17\right.$ th MO) to the virtual $\pi^{*}$ orbitals, which corresponds, to some extent, to 
TABLE III. Calculated excitation energies (in eV), oscillator strengths $f(r)$, and second moments for cyclopentadiene.

\begin{tabular}{|c|c|c|c|c|c|c|c|c|}
\hline \multirow[b]{2}{*}{ State } & \multirow[b]{2}{*}{ Nature $^{a}$} & \multirow[b]{2}{*}{$\Delta E^{\mathrm{b}}$} & \multirow[b]{2}{*}{$\mathrm{Pol}^{\mathrm{c}}$} & \multirow[b]{2}{*}{$f(r)$} & \multicolumn{4}{|c|}{ Second moment } \\
\hline & & & & & $\left\langle x^{2}\right\rangle$ & $\left\langle y^{2}\right\rangle$ & $\left\langle z^{2}\right\rangle$ & $\left\langle r^{2}\right\rangle$ \\
\hline $1{ }^{1} A_{1}$ & ground & 0.0 & & 0 & 25.51 & 22.02 & 20.93 & 68.46 \\
\hline $1{ }^{1} B_{2}$ & $V^{\mathrm{d}}$ & 5.54 & $y$ & 0.1305 & 26.92 & 21.43 & 24.00 & 72.35 \\
\hline $1{ }^{1} A_{2}$ & $3 s-R$ & 5.58 & & 0.0000 & 44.96 & 36.44 & 37.78 & 119.18 \\
\hline $1{ }^{1} B_{1}$ & $3 p_{y}-R$ & 6.17 & $x$ & 0.0270 & 38.98 & 71.87 & 35.02 & 145.87 \\
\hline $2{ }^{1} A_{2}$ & $3 p_{x}-R$ & 6.21 & & 0.0000 & 41.05 & 35.17 & 76.98 & 153.20 \\
\hline $2{ }^{1} B_{2}$ & $3 p_{z}-R$ & 6.22 & $y$ & 0.0311 & 75.85 & 32.90 & 34.13 & 142.88 \\
\hline $3{ }^{1} A_{2}$ & $3 d_{x x}-R$ & 6.69 & & 0.0000 & 51.40 & 71.26 & 36.07 & 158.73 \\
\hline $2{ }^{1} B_{1}$ & $3 d_{y z}-R$ & 6.71 & $x$ & 0.0003 & 38.39 & 65.04 & 67.72 & 171.15 \\
\hline $2{ }^{1} A_{1}$ & $V \sim$ mix $^{\mathrm{d}}$ & 6.76 & $z$ & 0.0009 & 30.88 & 27.03 & 23.69 & 81.60 \\
\hline $4{ }^{1} A_{2}$ & $3 d_{y y-z z}-R$ & 6.77 & & 0.0000 & 42.35 & 47.71 & 90.41 & 180.47 \\
\hline $3{ }^{1} B_{2}$ & $3 d_{x z}-R$ & 6.93 & $y$ & 0.0005 & 91.86 & 37.32 & 85.15 & 214.33 \\
\hline $3{ }^{1} A_{1}$ & $3 d_{x y}-R$ & 6.96 & $z$ & 0.0007 & 91.12 & 83.29 & 39.29 & 213.70 \\
\hline $5^{1} A_{2}$ & $4 s-R$ & 7.07 & & 0.0000 & 166.20 & 88.91 & 88.85 & 343.96 \\
\hline $3{ }^{1} B_{1}$ & $4 p_{y}-R$ & 7.24 & $x$ & 0.0072 & 98.90 & 245.10 & 92.76 & 436.76 \\
\hline $4{ }^{1} B_{2}$ & $4 p_{x}-R$ & 7.25 & $y$ & 0.0078 & 249.18 & 89.72 & 91.93 & 430.83 \\
\hline $6{ }^{1} A_{2}$ & $4 p_{z}-R$ & 7.27 & & 0.0000 & 104.17 & 96.81 & 262.05 & 463.03 \\
\hline $7{ }^{1} A_{2}$ & $4 d_{x x}-R$ & 7.42 & & 0.0000 & 142.36 & 192.34 & 129.62 & 464.32 \\
\hline $4{ }^{1} B_{1}$ & $4 d_{y z}-R$ & 7.46 & $x$ & 0.0002 & 83.37 & 198.85 & 200.45 & 482.67 \\
\hline $8{ }^{1} A_{2}$ & $4 d_{y y-z z}-R$ & 7.48 & & 0.0000 & 86.10 & 167.37 & 238.59 & 492.06 \\
\hline $5{ }^{1} B_{2}$ & $4 d_{x z}-R$ & 7.55 & $y$ & 0.0001 & 217.01 & 78.96 & 208.40 & 504.37 \\
\hline $4{ }^{1} A_{1}$ & $4 d_{x y}-R$ & 7.57 & $z$ & 0.0010 & 216.60 & 208.70 & 80.41 & 505.71 \\
\hline $9{ }^{1} A_{2}$ & $5 s-R$ & 7.60 & & 0.0000 & 382.32 & 249.15 & 248.35 & 879.82 \\
\hline $5{ }^{1} B_{1}$ & $5 p_{y}-R$ & 7.73 & $x$ & 0.0083 & 143.20 & 374.50 & 136.59 & 654.29 \\
\hline $6{ }^{1} B_{2}$ & $5 p_{x}-R$ & 7.74 & $y$ & 0.0054 & 402.27 & 140.63 & 396.33 & 686.23 \\
\hline $6{ }^{1} B_{1}$ & $3 s-R$ & 7.93 & $x$ & 0.0095 & 54.28 & 68.06 & 48.27 & 170.61 \\
\hline $7{ }^{1} B_{1}$ & $5 d_{y z}-R$ & 8.08 & $x$ & 0.0000 & 82.30 & 195.47 & 196.58 & 474.35 \\
\hline $5{ }^{1} A_{1}$ & $V \sim$ mix. $^{\mathrm{d}}$ & 8.17 & $z$ & 0.5377 & 61.92 & 54.32 & 35.78 & 152.02 \\
\hline $7{ }^{1} B_{2}$ & $5 d_{x z}-R$ & 8.23 & $y$ & 0.0000 & 188.92 & 69.64 & 181.80 & 440.36 \\
\hline $6{ }^{1} A_{1}$ & $5 d_{x y}-R$ & 8.27 & $z$ & 0.0966 & 155.70 & 144.22 & 61.47 & 361.39 \\
\hline $8{ }^{1} B_{1}$ & $3 p_{z}^{\prime}-R$ & 8.47 & $x$ & 0.0122 & 37.81 & 38.86 & 66.97 & 143.64 \\
\hline $7{ }^{1} A_{1}$ & $3 p_{x}^{\prime}-R$ & 8.56 & $z$ & 0.0216 & 78.38 & 41.77 & 34.09 & 154.24 \\
\hline $9{ }^{1} B_{1}$ & $4 p_{z}^{\prime}-R$ & 8.59 & $x$ & 0.0038 & 34.11 & 48.24 & 71.26 & 138.81 \\
\hline $8^{1} B_{2}$ & $\pi-\sigma^{*}$ & 9.07 & $y$ & 0.0248 & 153.34 & 58.13 & 58.81 & 270.28 \\
\hline $8{ }^{1} A_{1}$ & $3 d_{x z}^{\prime}-R$ & 9.23 & $z$ & 0.0290 & 95.53 & 40.91 & 85.19 & 221.63 \\
\hline $9{ }^{1} B_{2}$ & $3 d_{x y}^{\prime}-R$ & 9.25 & $y$ & 0.0053 & 83.56 & 75.79 & 38.17 & 197.52 \\
\hline $10{ }^{1} B_{2}$ & $4 d_{x y}^{\prime}-R$ & 9.43 & $y$ & 0.0104 & 63.91 & 54.32 & 41.16 & 159.39 \\
\hline $9{ }^{1} A_{1}$ & $4 p_{x}^{\prime y}-R$ & 9.54 & $z$ & 0.0016 & 249.88 & 92.56 & 91.76 & 434.20 \\
\hline $1{ }^{3} B_{2}$ & $V^{\mathrm{d}}$ & 3.40 & & 0.0 & 25.61 & 21.09 & 22.72 & 69.42 \\
\hline $1{ }^{3} A_{1}$ & $V^{\mathrm{d}}$ & 5.18 & & 0.0 & 25.70 & 22.40 & 22.08 & 70.18 \\
\hline $1{ }^{3} A_{2}$ & $3 s-R$ & 5.56 & & 0.0 & 45.35 & 34.80 & 37.92 & 118.07 \\
\hline $1{ }^{3} B_{2}$ & $3 p_{y}-R$ & 6.19 & & 0.0 & 40.47 & 75.77 & 36.42 & 152.66 \\
\hline
\end{tabular}

${ }^{\mathrm{a}} V$ and $R$ denote, respectively, valence and Rydberg excited state.

${ }^{\mathrm{b}}$ Transition energy.

${ }^{c}$ Polarization direction of transition moment.

${ }^{\mathrm{d}}$ See text.

Robin's superposition of the $N \rightarrow V_{2}\left(\widetilde{X}^{1} A_{1} \rightarrow{ }^{1} A_{1}\right)$ and $N$ $\rightarrow V_{3}\left(\widetilde{X}^{1} A_{1} \rightarrow{ }^{1} A_{1}\right)$ excitations. From this perspective, our current results support Robin's suggestion.

In the higher energy region, several transitions are observed in peak IV located at $8.7-9.4 \mathrm{eV}$ of the spectrum shown in Fig. 2. The present SAC-CI calculations show that the excitation energies in this region were relatively basis set-dependent, and place $8{ }^{1} B_{2}, 8{ }^{1} A_{1}, 9{ }^{1} B_{2}, 10{ }^{1} B_{2}$, and $9^{1} A_{1}$ states at 9.07, 9.23, 9.25, 9.43, and $9.54 \mathrm{eV}$ with intensities of $0.0248,0.0290,0.0053,0.0104$, and 0.0016 , respectively. McDiarmid and Sabljic ${ }^{27}$ experimentally assigned peak IV observed at $9.2 \mathrm{eV}$ to the $N \rightarrow V_{4}\left(\widetilde{X}^{1} A_{1} \rightarrow{ }^{1} B_{2}\right)$ valence excitation. RPA calculations gave the second ${ }^{1} B_{2}$ state at $10.46 \mathrm{eV}$ with an oscillator strength of 0.0901. CASPT2 and MRMP gave no excited states in this region.

Finally, we discuss the valence excited state $2{ }^{1} A_{1}$. This state was not found in the spectra. Our present calculations gave the $2{ }^{1} A_{1}$ state at $6.76 \mathrm{eV}$ with a very weak oscillator strength of 0.0009 and a second moment of 81.60 a.u. ${ }^{2}$, which clearly defines it as a valence excited state. Its main configuration was almost purely composed of single excitations, 17 th $\mathrm{MO} \rightarrow 48$ th $\mathrm{MO}$ and 18 th $\mathrm{MO} \rightarrow 54$ th $\mathrm{MO}$, and there was little double-excitation mixing in this state. CASPT2 gave a value of $6.31 \mathrm{eV}$ with an intensity of 0.0003 and its nature was reported to be a mixture of the configurations $2 b_{1} \rightarrow 3 b_{1}$ and $1 a_{2} \rightarrow 2 a_{2}$ and the double excitation of $0.43\left[\left(1 a_{2}\right)^{2} \rightarrow\left(3 b_{1}\right)^{2}\right]$. MRMP gave $6.42 \mathrm{eV}$ with an intensity of 0.0039 , and its nature was almost the same as that by 
TABLE IV. SAC-CI results compared with the experimental excitation energies (in eV) and other theoretical results for cyclopentadiene.

\begin{tabular}{|c|c|c|c|c|c|c|c|c|c|c|}
\hline \multirow[b]{2}{*}{ State } & \multirow[b]{2}{*}{ Nature $^{\mathrm{a}}$} & \multicolumn{2}{|c|}{ SAC-CI } & \multirow[b]{2}{*}{ Expt. $^{c}$} & \multicolumn{3}{|c|}{$\mathrm{CASPT}^{\mathrm{d}}$} & \multicolumn{3}{|c|}{$\mathrm{MRMP}^{\mathrm{e}}$} \\
\hline & & $f(r)$ & $\Delta E^{\mathrm{b}}$ & & $\Delta E$ & $f(r)$ & State & $\Delta E$ & $f(r)$ & State \\
\hline $1^{1} A_{1}$ & ground & 0 & 0.0 & & & & & & & \\
\hline${ }^{1}{ }^{1} B_{2}$ & $V^{\mathrm{f}}$ & 0.1305 & 5.54 & $5.26,5.34$ & 5.27 & 0.1475 & $1{ }^{1} B_{2}$ & 5.19 & 0.1460 & $1^{1} B_{2}$ \\
\hline $1^{1} A_{2}$ & $3 s-R$ & forbidden & 5.58 & 5.68 & 5.65 & forbidden & $1^{1} A_{2}$ & 5.62 & forbidden & $1{ }^{1} A_{2}$ \\
\hline $1{ }^{1} B_{1}$ & $3 p_{y}-R$ & 0.0270 & 6.17 & 6.25 & 6.24 & 0.0306 & ${ }^{1}{ }^{1} B_{1}$ & 6.24 & 0.0174 & ${ }^{1}{ }^{1} B_{1}$ \\
\hline $2{ }^{1} A_{2}$ & $3 p_{x}-R$ & forbidden & 6.21 & 6.26 & 6.30 & forbidden & $2{ }^{1} A_{2}$ & 6.27 & forbidden & $2{ }^{1} A_{2}$ \\
\hline $2{ }^{1} B_{2}$ & $3 p_{z}-R$ & 0.0311 & 6.22 & 6.31 & 6.25 & 0.0248 & $2{ }^{1} B_{2}$ & 6.25 & 0.0149 & $2{ }^{1} B_{2}$ \\
\hline $3{ }^{1} A_{2}$ & $3 d_{x x}-R$ & forbidden & 6.69 & & 6.85 & forbidden & $3{ }^{1} A_{2}$ & 6.81 & forbidden & $3{ }^{1} A_{2}$ \\
\hline $2{ }^{1} B_{1}$ & $3 d_{y z}-R$ & 0.0003 & 6.71 & & 6.87 & 0.0003 & $2{ }^{1} B_{1}$ & 6.86 & 0.0000 & $2{ }^{1} B_{1}$ \\
\hline $2{ }^{1} A_{1}$ & $V \sim \operatorname{mix} .^{f}$ & 0.0009 & 6.76 & & 6.31 & 0.0003 & $2{ }^{1} A_{1}$ & 6.42 & 0.0039 & $2{ }^{1} A_{1}$ \\
\hline $4^{1} A_{2}$ & $3 d_{y y-z z}-R$ & forbidden & 6.77 & & 6.91 & forbidden & $4^{1} A_{2}$ & 6.88 & forbidden & $4{ }^{1} A_{2}$ \\
\hline $3{ }^{1} B_{2}$ & $3 d_{x z}-R$ & 0.0005 & 6.93 & 6.80 & 6.86 & 0.0101 & $3{ }^{1} B_{2}$ & 6.91 & 0.0105 & $3{ }^{1} B_{2}$ \\
\hline $3{ }^{1} A_{1}$ & $3 d_{x y}-R$ & 0.0007 & 6.96 & & 6.93 & 0.0208 & $3{ }^{1} A_{1}$ & 6.89 & 0.0003 & $3{ }^{1} A_{1}$ \\
\hline $5{ }^{1} A_{2}$ & $4 s-R$ & forbidden & 7.07 & & & & & & & \\
\hline $3{ }^{1} B_{1}$ & $4 p_{y}-R$ & 0.0072 & 7.24 & 7.05 & & & & & & \\
\hline $4{ }^{1} B_{2}$ & $4 p_{x}-R$ & 0.0078 & 7.25 & & & & & & & \\
\hline $6^{1} A_{2}$ & $4 p_{z}-R$ & forbidden & 7.27 & & & & & & & \\
\hline $7^{1} A_{2}$ & $4 d_{x x}-R$ & forbidden & 7.42 & & & & & & & \\
\hline $4{ }^{1} B_{1}$ & $4 d_{y z}-R$ & 0.0002 & 7.46 & & & & & & & \\
\hline $8^{1} A_{2}$ & $4 d_{y y-z z}-R$ & forbidden & 7.48 & & & & & & & \\
\hline $5^{1} B_{2}$ & $4 d_{x z}-R$ & 0.0001 & 7.55 & & & & & & & \\
\hline $4{ }^{1} A_{1}$ & $4 d_{x y}-R$ & 0.0010 & 7.57 & & & & & & & \\
\hline $9{ }^{1} A_{2}$ & $5 s-R$ & forbidden & 7.60 & & & & & & & \\
\hline $5{ }^{1} B_{1}$ & $5 p_{y}-R$ & 0.0083 & 7.73 & 7.61 & & & & & & \\
\hline $6{ }^{1} B_{2}$ & $5 p_{x}-R$ & 0.0054 & 7.74 & & & & & & & \\
\hline $6{ }^{1} B_{1}$ & $3 s-R$ & 0.0095 & 7.93 & 7.84 & & & & 7.80 & 0.0217 & $3{ }^{1} B_{1}$ \\
\hline $7{ }^{1} B_{1}$ & $5 d_{y z}-R$ & 0.0000 & 8.08 & 8.03 & 7.95 & 0.0252 & $3{ }^{1} B_{1}$ & & & \\
\hline $5^{1} A_{1}$ & $V \sim \operatorname{mix} .^{\mathrm{f}}$ & 0.5377 & 8.17 & $7.88,8.06$ & 7.89 & 0.4415 & $4{ }^{1} A_{1}$ & 8.11 & 0.5020 & $4{ }^{1} A_{1}$ \\
\hline $7{ }^{1} B_{2}$ & $5 d_{x z}-R$ & 0.0000 & 8.23 & 8.19 & & & & & & \\
\hline $6{ }^{1} A_{1}$ & $5 d_{x y}-R$ & 0.0966 & 8.27 & 8.28 & & & & & & \\
\hline $8{ }^{1} B_{1}$ & $3 p_{z}^{\prime}-R$ & 0.0122 & 8.47 & 8.36 & & & & & & \\
\hline $7{ }^{1} A_{1}$ & $3 p_{x}^{\prime}-R$ & 0.0216 & 8.56 & & & & & & & \\
\hline $9{ }^{1} B_{1}$ & $4 p_{z}^{\prime}-R$ & 0.0038 & 8.59 & & & & & & & \\
\hline $8{ }^{1} B_{2}$ & $\pi-\sigma^{*}$ & 0.0248 & 9.07 & & & & & & & \\
\hline $8^{1} A_{1}$ & $3 d_{x z}^{\prime}-R$ & 0.0290 & 9.23 & 9.2 & & & & & & \\
\hline $9{ }^{1} B_{2}$ & $3 d_{x y}^{\prime}-R$ & 0.0053 & 9.25 & & & & & & & \\
\hline $10^{1} B_{2}$ & $4 d_{x y}^{\prime}-R$ & 0.0104 & 9.43 & & & & & & & \\
\hline $9{ }^{1} A_{1}$ & $4 p_{x}^{\prime}-R$ & 0.0016 & 9.54 & & & & & & & \\
\hline $1^{3} B_{2}$ & $V^{\mathrm{f}}$ & 0.0 & 3.40 & 3.10 & 3.15 & & & & & \\
\hline $1{ }^{3} A_{1}$ & $V^{\mathrm{f}}$ & 0.0 & 5.18 & $>4.7$ & 4.90 & & & & & \\
\hline $1{ }^{3} A_{2}$ & $3 s-R$ & 0.0 & 5.56 & & 5.63 & & & & & \\
\hline $1^{3} B_{2}$ & $3 p_{y}-R$ & 0.0 & 6.19 & & 6.2 & & & & & \\
\hline
\end{tabular}

${ }^{\mathrm{a}} V$ and $R$ denote, respectively, valence and Rydberg excited state.

${ }^{\mathrm{b}}$ Transition energy.

${ }^{\mathrm{c}}$ References 4 and 26.

${ }^{\mathrm{d}}$ Reference 8.
${ }^{\mathrm{e}}$ Reference 9.
${ }^{\mathrm{f}}$ See text.
CASPT2 except that Rydberg mixing was found. The reason for this difference between our result and other theoretical results is unclear. This excited state is not observed experimentally because of the weak oscillator strength, so it is difficult to make a conclusion experimentally. However, in the case of thiophene, which will be discussed in a later paper, ${ }^{28}$ the $2{ }^{1} A_{1}$ state has been well reproduced by the SAC-CI SD-R method with regard to both excitation energy and oscillator strength, in comparison with the experimental data in the VUV spectrum.

\section{B. Singlet Rydberg states}

The lowest singlet Rydberg transition located at $5.63 \mathrm{eV}$ with a rather weak feature was assigned experimentally by
Robin $^{26}$ and McDiarmid and Sabljic ${ }^{27}$ to the symmetryforbidden $1{ }^{1} A_{2}\left(1 a_{2} \rightarrow 3 s\right)$ state. In the present calculations, the $1{ }^{1} A_{2}$ state was calculated at $5.58 \mathrm{eV}$. In the two-photon resonant multiphoton ionization (2RMPI) spectrum of Sabljic et $a .^{29}$ the group of transition which occurred in the region $6.0-6.4 \mathrm{eV}$ was assigned to the $1 a_{2} \rightarrow 3 p$ Rydberg series, $6.25\left(3 p_{y}\right), 6.26\left(3 p_{z}\right)$, and $6.31\left(3 p_{x}\right) \mathrm{eV}$. The present results for these states are $6.17 \mathrm{eV}\left(1{ }^{1} B_{1}: 3 p_{y}\right), 6.21 \mathrm{eV}$ $\left(2{ }^{1} A_{2}: 3 p_{x}\right)$, and $6.22 \mathrm{eV}\left(2{ }^{1} B_{2}: 3 p_{z}\right)$ in a slightly different order. Note that the splitting of the $3 p$ series of Rydberg is very small in both experimental and theoretical results.

Frueholz et al. ${ }^{4}$ also observed some apparent Rydberg transitions at 6.80, 7.05, 7.61, 7.84, 8.03, 8.19, $9.2 \mathrm{eV}$ and a higher energy region, but these states were not experimen- 


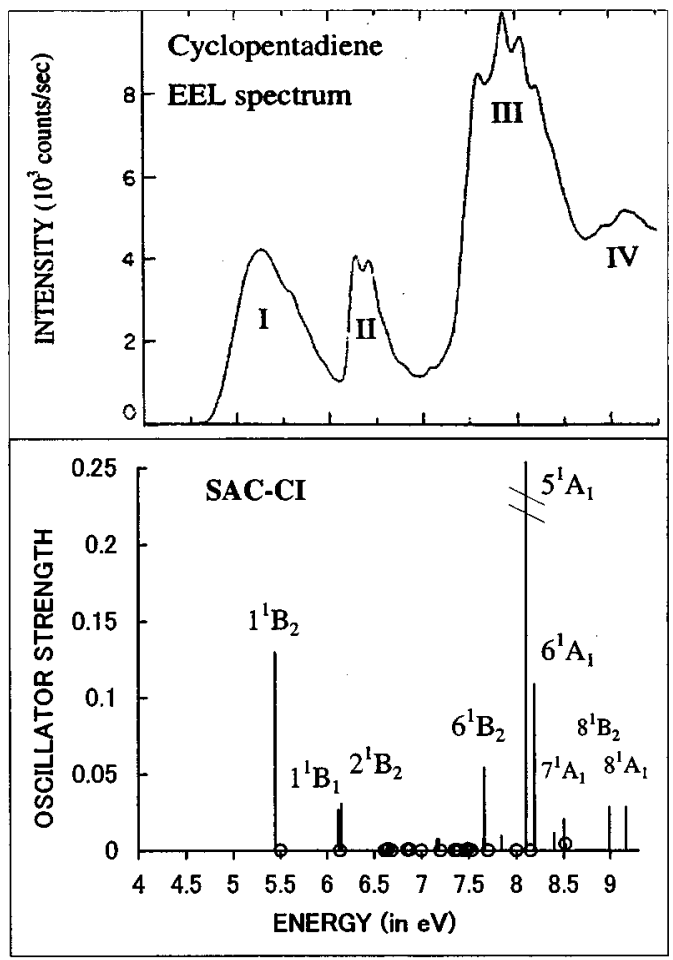

FIG. 2. EEL spectrum and calculated excitation energies and oscillator strengths of cyclopentadiene.

tally assigned. Our current SAC-CI calculations gave a large number of Rydberg excitations, as shown in Tables III and IV. The $3{ }^{1} B_{2}$ state $1 a_{2} \rightarrow 3 d_{x z}$ transition was calculated at $6.93 \mathrm{eV}$ with an intensity of 0.0005 , the $3{ }^{1} A_{1}$ state $1 a_{2}$ $\rightarrow 3 d_{x y}$ was at $6.96 \mathrm{eV}$ with an intensity of 0.0007 , and they were both assigned to the $6.80 \mathrm{eV}$ region in the spectrum. The $3{ }^{1} B_{1}$ state $1 a_{2} \rightarrow 4 p_{y}$ transition at $7.24 \mathrm{eV}$ with an intensity of 0.0072 and the $4{ }^{1} B_{2}$ state $1 a_{2} \rightarrow 4 p_{x}$ at $7.25 \mathrm{eV}$ with an intensity of 0.0078 were both assigned to the $7.05 \mathrm{eV}$ region of the spectrum. In addition, in the higher energy region of 7.07-7.74 eV we obtained many Rydberg states as seen from Table IV, but these states were not reported in the CASPT2 and MRMP studies. We assigned the transition observed at the $7.84 \mathrm{eV}$ region to the $6{ }^{1} B_{1} \quad 2 b_{1} \rightarrow 3 s$ state (denoted as $3 s^{\prime}$ to distinguish the $1 a_{2} \rightarrow 3 s$ series, for convenience the convention is reserved in thereafter) that was calculated at $7.93 \mathrm{eV}$ with an intensity of 0.0095 , where the space symmetry is the same as in the MRMP assignment, $3{ }^{1} B_{1}$. The transition observed in the $8.03 \mathrm{eV}$ region is assigned to the $7{ }^{1} B_{1}\left(5 d_{y z}\right)$ state calculated at $8.08 \mathrm{eV}$. In the even higher energy region, our assignments are shown in Table IV. Both CASPT2 and MRMP assigned the peaks at 6.80 and $7.05 \mathrm{eV}$ to the $1 a_{2} \rightarrow 3 d$ transitions, but CASPT2 assigned the $8.03 \mathrm{eV}$ peak to the $2 b_{1} \rightarrow 3 s$ transition, while MRMP assigned this transition to the peak at $7.84 \mathrm{eV}$; these assignments were not consistent.

\section{Triplet excited states}

The lowest-energy transition of CP observed by electron impact spectroscopy ${ }^{4}$ had an effective Franck-Condon region extending from 2.5 to $3.9 \mathrm{eV}$ with an intensity maxi-

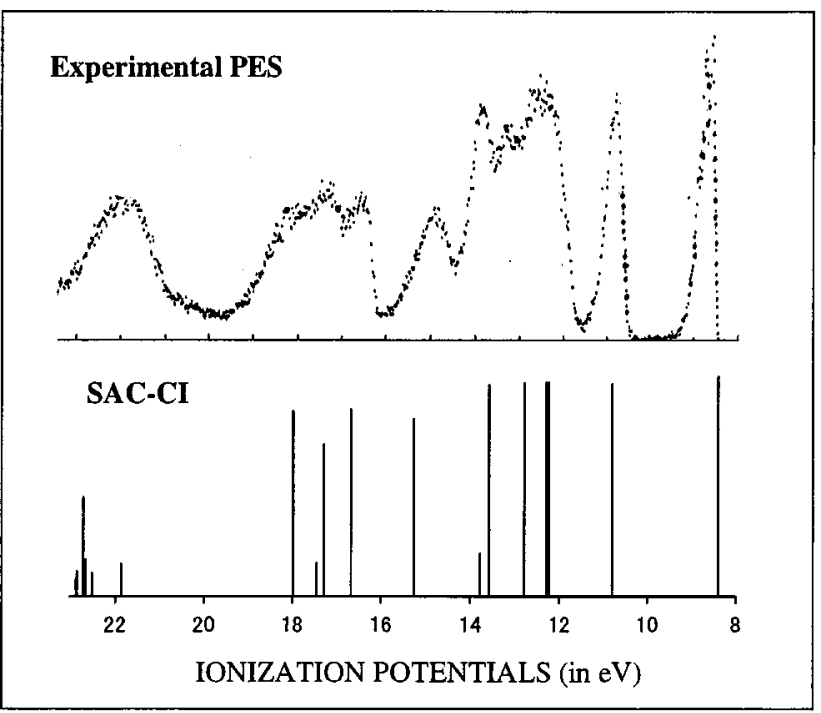

FIG. 3. PES spectrum and calculated ionization energies and monopole intensities of cyclopentadiene.

mum at $3.10 \mathrm{eV}$, which was similar to that obtained by Evans $^{30}$ using the oxygen perturbation technique. Frueholz et $a .^{4}{ }^{4}$ experimentally assigned this transition to the singlettriplet excitation, $N \rightarrow T_{1}\left(\widetilde{X}^{1} A_{1} \rightarrow{ }^{3} B_{2}\right)$. Our current calculations gave the $1^{3} B_{2}$ state at $3.40 \mathrm{eV}$ and its second moment was 69.42, which clearly showed it as a valence excited state. Its main configuration is ${ }^{3} \pi-\pi^{*}$ excitation of 18 $\rightarrow 48$.

The second triplet state has not yet been observed by spectroscopic studies. Frueholz et al. ${ }^{4}$ suggested that it was hidden behind the singlet valence excitation at $5.2 \mathrm{eV}$ and estimated the excitation energy of the second triplet state to be above $4.7 \mathrm{eV}$. Our current results predict this state to be $1^{3} A_{1}$ at $5.18 \mathrm{eV}$ with a second moment of 70.18 , which means it is a valence-excited state. Its main configuration is a mixture of the ${ }^{3} \pi-\pi^{*}$ excitation of $17 \rightarrow 48$ and $18 \rightarrow 54$. CASPT2 also computed these two valence excited states at $3.15\left(1{ }^{3} B_{2}\right)$ and $4.90 \mathrm{eV}\left(1{ }^{3} A_{1}\right)$. In addition, the lowest ${ }^{3} A_{2}$ and ${ }^{3} B_{1}$ states have also been calculated at 5.56 and 6.19 $\mathrm{eV}$, respectively, and these are Rydberg excitations in nature as seen from Table IV.

\section{Ionized states}

The electronic structure of the ground state of $\mathrm{CP}$ can be described with 18 molecular orbitals (MOs). They are given in Table II together with their natures and symmetries.

A photoelectron spectrum (PES) of $\mathrm{CP}$ has been measured by Derrick et al. ${ }^{5}$ up to $23 \mathrm{eV}$, containing both outerand inner-valence regions: it is illustrated in the upper side of Fig. 3. Table V shows the SAC-CI general-R theoretical binding energies, monopole intensities, main configurations, and assignments of the ionization peaks in PES. Table $\mathrm{V}$ also shows the SAC-CI SD-R results and Koopmans values. The SAC-CI general-R method gave satisfactory results, which were better than the SD-R results. The Koopmans values derivate strongly from the experimental values except for those of the two lowest states. In Fig. 3, the experimental 
TABLE V. Calculated ionization potentials, Koopmans energies, and experimental values of PES for cyclopentadiene.

\begin{tabular}{|c|c|c|c|c|c|c|c|}
\hline \multirow[b]{2}{*}{ State } & \multicolumn{3}{|c|}{ SAC-CI General-R } & \multirow{2}{*}{$\frac{\text { PES (Ref. 5) }}{\operatorname{IP}(\mathrm{eV})}$} & \multicolumn{2}{|c|}{ SAC-CI SD-R } & \multirow{2}{*}{$\begin{array}{c}\text { Koopmans } \\
\text { energies }\end{array}$} \\
\hline & Main configuration (MC) & $\mathrm{IP}(\mathrm{eV})$ & Intensity $^{\mathrm{a}}$ & & $\mathrm{IP}(\mathrm{eV})$ & $\mathrm{MC}$ & \\
\hline $1^{2} A_{2}$ & $0.95^{\mathrm{b}}(18 \rightarrow \infty)$ & 8.39 & 0.90 & 8.566 & 8.30 & $0.98(18 \rightarrow \infty)$ & $8.37\left(1 a_{2}\right)$ \\
\hline $1^{2} B_{1}$ & $0.93(17 \rightarrow \infty)$ & 10.80 & 0.87 & 10.724 & 10.53 & $0.97(17 \rightarrow \infty)$ & $11.24\left(2 b_{1}\right)$ \\
\hline $1^{2} B_{2}$ & $0.93(16 \rightarrow \infty)$ & 12.23 & 0.87 & $\sim 12.2$ & 11.97 & $0.96(16 \rightarrow \infty)$ & $13.62\left(6 b_{2}\right)$ \\
\hline $1^{2} A_{1}$ & $0.93(15 \rightarrow \infty)$ & 12.29 & 0.87 & $\sim 12.6$ & 11.95 & $0.96(15 \rightarrow \infty)$ & $13.79\left(9 a_{1}\right)$ \\
\hline $2{ }^{2} A_{1}$ & $0.93(14 \rightarrow \infty)$ & 12.79 & 0.87 & 13.2 & 12.50 & $0.96(14 \rightarrow \infty)$ & $14.39\left(8 a_{1}\right)$ \\
\hline $2^{2} B_{2}$ & $0.93(13 \rightarrow \infty)$ & 13.58 & 0.86 & 13.8 & 13.33 & $0.96(13 \rightarrow \infty)$ & $15.29\left(5 b_{2}\right)$ \\
\hline $2^{2} B_{1}$ & $0.39(12 \rightarrow \infty), 0.99(18 \rightarrow \infty ; 18 \rightarrow 48)$ & 13.78 & 0.18 & & 15.91 & $0.40(12 \rightarrow \infty)^{\mathrm{g}}$ & \\
\hline $3^{2} B_{1}$ & $0.85(12 \rightarrow \infty), 0.46(18 \rightarrow \infty ; 18 \rightarrow 48)$ & 15.26 & 0.72 & 14.8 & & & $16.07\left(1 b_{1}\right)$ \\
\hline $3{ }^{2} A_{1}$ & $0.87(11 \rightarrow \infty), 0.23(18 \rightarrow \infty ; 16 \rightarrow 48)$ & 16.67 & 0.76 & 16.4 & 16.57 & $0.93(11 \rightarrow \infty)$ & $18.94\left(7 a_{1}\right)$ \\
\hline $3^{2} B_{2}$ & $0.77(10 \rightarrow \infty), 0.41(18 \rightarrow \infty ; 15 \rightarrow 48)$ & 17.28 & 0.62 & 17.5 & 17.23 & $0.93(10 \rightarrow \div)$ & $19.81\left(4 b_{2}\right)$ \\
\hline $4^{2} A_{1}$ & $0.13(11 \rightarrow \infty), 0.87(18 \rightarrow \infty ; 16 \rightarrow 48)$ & 17.43 & 0.02 & & & & \\
\hline $4^{2} B_{2}$ & $0.40(10 \rightarrow \infty), 0.80(18 \rightarrow \infty ; 15 \rightarrow 48)$ & 17.44 & 0.14 & & & & \\
\hline $5^{2} A_{1}$ & $0.85(9 \rightarrow \infty), 0.20(18 \rightarrow \infty ; 16 \rightarrow 48)$ & 17.98 & 0.76 & 18.4 & 17.89 & $0.93(9 \rightarrow \infty)$ & $20.57\left(6 a_{1}\right)$ \\
\hline $5^{2} B_{2}$ & $\begin{array}{l}0.87(18 \rightarrow \infty ; 14 \rightarrow 48)+0.39(14 \rightarrow \infty \\
18 \rightarrow 48)-0.31(18 \rightarrow \infty ; 14 \rightarrow 63)\end{array}$ & 18.07 & 0.03 & & & & \\
\hline $11^{2} A_{1}$ & $\begin{array}{l}0.65(16 \rightarrow \infty ; 18 \rightarrow 48)+0.21(16 \rightarrow \infty \\
18 \rightarrow 63)-0.20(15 \rightarrow \infty ; 18 \rightarrow 67)\end{array}$ & 19.70 & 0.07 & & & & \\
\hline $13^{2} B_{2}$ & $\begin{array}{l}-0.72(14 \rightarrow \infty ; 14 \rightarrow 48)+0.24(14 \rightarrow \infty \\
18 \rightarrow 63)+0.23(13 \rightarrow \infty ; 17 \rightarrow 48)\end{array}$ & 20.36 & 0.04 & & & & \\
\hline $15^{2} A_{1}$ & $\begin{array}{l}-0.60(13 \rightarrow \infty ; 18 \rightarrow 48)+0.31(14 \rightarrow \infty \\
17 \rightarrow 63)+0.29(16 \rightarrow \infty ; 18 \rightarrow 48)\end{array}$ & 20.89 & 0.05 & & & & \\
\hline $18^{2} B_{2}$ & $\begin{array}{l}-0.46(16 \rightarrow \infty ; 17 \rightarrow 48)+0.35(16 \rightarrow \infty \\
18 \rightarrow 67)-0.27(15 \rightarrow \infty ; 18 \rightarrow 48)\end{array}$ & 21.47 & 0.06 & & & & \\
\hline $21^{2} B_{2}$ & $0.36(7 \rightarrow \infty), 0.37(18 \rightarrow \infty ; 18 \rightarrow 58)^{\mathrm{c}}$ & 21.86 & 0.13 & 22.0 & & & $26.00\left(5 a_{1}\right)$ \\
\hline $24^{2} B_{2}$ & $\begin{array}{l}-0.31(7 \rightarrow \infty), 0.58(18 \rightarrow \infty ; 18 \rightarrow 31), 0.43 \\
(18 \rightarrow \infty ; 18 \rightarrow 51)\end{array}$ & 22.51 & 0.10 & & & & \\
\hline $25^{2} \mathrm{~B}_{2}$ & $0.39(7 \rightarrow \infty), 0.60(18 \rightarrow \infty ; 18 \rightarrow 34)^{\mathrm{e}}$ & 22.65 & 0.15 & & & & \\
\hline $29^{2} A_{1}$ & $0.63(8 \rightarrow \infty), 0.29(18 \rightarrow \infty ; 18 \rightarrow 33)^{\mathrm{d}}$ & 22.70 & 0.41 & 22.3 & & & $26.30\left(3 b_{2}\right)$ \\
\hline $28{ }^{1} B_{2}$ & $0.32(7 \rightarrow \infty), 0.37(18 \rightarrow \infty ; 18 \rightarrow 34)^{\mathrm{f}}$ & 22.84 & 0.10 & & & & \\
\hline $29^{2} \mathrm{~B}_{2}$ & $\begin{array}{l}0.27(7 \rightarrow \infty), 0.40(18 \rightarrow \infty ; 18 \rightarrow 34), 0.27 \\
(18 \rightarrow \infty ; 18 \rightarrow 25)\end{array}$ & 22.87 & 0.07 & & & & \\
\hline
\end{tabular}

${ }^{a}$ Monopole intensity including the final state and initial state configuration interactions. The state whose intensity larger than 0.01 is given.

${ }^{\mathrm{b}}$ The main configuration whose absolute value of the coefficient larger than 0.2 is given.

${ }^{\mathrm{c}}$ Also includes configurations: $0.31(18 \rightarrow \infty ; 18 \rightarrow 66)$.

${ }^{\mathrm{d}}$ Also includes configurations: $0.25(18 \rightarrow \infty ; 18 \rightarrow 59)$.

${ }^{\mathrm{e}}$ Also includes configurations: $0.38(18 \rightarrow \infty ; 18 \rightarrow 56)$.

${ }^{\mathrm{f}}$ Also includes configurations: $0.25(17 \rightarrow \infty ; 18 \rightarrow 29)$.

${ }^{\mathrm{g}}$ Also includes configurations: $1.00(18 \rightarrow 48+18 \rightarrow \infty)$ and $0.38(18 \rightarrow \infty ; 18 \rightarrow 63)$.

PES and the present theoretical spectrum calculated by the SAC-CI general-R method are compared.

The peaks at 8.566 and $10.724 \mathrm{eV}$ were experimentally assigned $^{5}$ to the outer two $\pi$ MOs $1 a_{2}$ and $2 b_{1}$. Our present SAC-CI general-R calculations computed these two states at 8.39 and $10.80 \mathrm{eV}$, respectively. It is clear from the main configurations (shown in Table IV) of the two ionized states $1^{2} A_{2}$ and $1{ }^{2} B_{1}$ that they are dominated by one-electron process, confirming the previously experimental assignment. The next sequential peaks at $\sim 12.2, \sim 12.6,13.2$, and 13.8 $\mathrm{eV}$ in PES were assigned to the $1^{2} B_{2}, 1^{2} A_{1}, 2^{2} A_{1}$, and $2^{2} B_{2}$ states calculated at $12.23,12.29,12.79$, and $13.58 \mathrm{eV}$, respectively, by the SAC-CI general-R method. These ionizations are also dominated by one-electron process, as seen from their main configurations. Note that the experimental peaks at $\sim 12.2$ and $\sim 12.6 \mathrm{eV}$ are overlapping ones with complicated vibrational structure.

In the energy region higher than $14.3 \mathrm{eV}$, other six sequential ionization peaks were observed at 14.8, 16.4, 17.5, $18.4,22.0$, and $22.3 \mathrm{eV}$. Our present SAC-CI general-R results calculations showed that these six ionizations were more or less characterized by multielectron processes. The peak at $14.8 \mathrm{eV}$ was assigned to the $3{ }^{2} B_{1}$ state calculated at $15.26 \mathrm{eV}$ with the main configurations that are a mixture of the single- and double-electron processes, $0.85(12 \rightarrow \infty)$ and $0.46(18 \rightarrow 48,18 \rightarrow \infty)$, respectively. The peak at $16.4 \mathrm{eV}$ was assigned to the $3{ }^{2} A_{1}$ state calculated at $16.67 \mathrm{eV}$ with the main configurations of $0.87(11 \rightarrow \infty), \quad 0.11(9 \rightarrow \infty)$, and $0.23(18 \rightarrow 48,16 \rightarrow \infty)$. The peak at $17.5 \mathrm{eV}$ was assigned to the $3{ }^{2} B_{2}$ state calculated at $17.28 \mathrm{eV}$ with the main configurations of $0.77(10 \rightarrow \infty)$ and $0.41(15 \rightarrow 48,18 \rightarrow \infty)$. The peak at $18.4 \mathrm{eV}$ was assigned to the $5^{2} A_{1}$ state calculated at 17.98 $\mathrm{eV}$ with the main configurations of $0.87(9 \rightarrow \infty), 0.17(11$ $\rightarrow \infty)$, and $0.19(18 \rightarrow 48,16 \rightarrow \infty)$. The peaks at about 22.0 and $22.3 \mathrm{eV}$ lie in a broad peak in PES. ${ }^{5}$ Our present SAC-CI general-R calculations gave many ionized states with the natures of the multielectron process in this region. The $29^{2} A_{1}$ state calculated at $22.70 \mathrm{eV}$ with a monopole intensity of 0.41 has the main configurations of $0.63(8 \rightarrow \infty), 0.29(18$ $\rightarrow 33,18 \rightarrow \infty)$, and $0.25(18 \rightarrow 59,18 \rightarrow \infty)$. The $21^{2} B_{2}$ state calculated at $21.86 \mathrm{eV}$ with a monopole intensity of 0.13 has the main configurations of $0.36(7 \rightarrow \infty), 0.37(18 \rightarrow 58,18$ 
$\rightarrow \infty)$, and $0.31(18 \rightarrow 66,18 \rightarrow \infty)$. The $25^{2} B_{2}$ state calculated at $22.51 \mathrm{eV}$ with a monopole intensity of 0.10 has the main configurations of $0.31(7 \rightarrow \infty), 0.58(18 \rightarrow 31,18 \rightarrow \infty)$, and $0.43(18 \rightarrow 51,18 \rightarrow \infty)$. The $26^{2} B_{2}$ state calculated at $22.65 \mathrm{eV}$ with a monopole intensity of 0.15 has the main configurations of $0.39(7 \rightarrow \infty), 0.60(18 \rightarrow 34,18 \rightarrow \infty)$, and $0.38(18 \rightarrow 56,18 \rightarrow \infty)$. The $28^{2} B_{2}$ state calculated at 22.84 $\mathrm{eV}$ with a monopole intensity of 0.10 has the main configurations of $0.32(7 \rightarrow \infty), 0.37(18 \rightarrow 34,18 \rightarrow \infty)$, and $0.25(18$ $\rightarrow 29,17 \rightarrow \infty)$. Thus our results show that the peaks at around $22.0 \mathrm{eV}$ consist of a series of correlation peaks.

Note that the present SAC-CI SD-R results (shown in Table V) are generally in agreement with the experimental PES data except for the three ionization peaks at 14.8, 22.0, and $22.3 \mathrm{eV}$, which are dominated by multiple-electron processes. Since the SD-R calculations did not include higherorder excitation operators, the ionization potentials of the inner-valence region were not accurately computed. A comparison of the general-R results with the SD-R results obviously shows that the inclusion of a small number of but important higher-order excitation operators by the SAC-CI general-R method remarkably improves the description of the binding energies in the inner-valence region.

\section{CONCLUSIONS}

In the present study, singlet and triplet excited states and ionized states of cyclopentadiene (CP) were reinvestigated by the SAC/SAC-CI method with an adequately extended basis set and a large active orbital space. The 40 low-lying singlet and triplet excited states were calculated and a reliable assignment for the UV and EEL spectra up to $8 \mathrm{eV}$ was made using the calculated excitation energies and oscillator strengths. Valence and Rydberg excited states were discussed separately in detail. Furthermore, the SAC-CI general-R method with the combined use of the exponential generation and perturbation selection schemes for including higher-order excitation operators was applied to the ionized states of CP up to $23 \mathrm{eV}$. A comparison of the experimental PES spectrum and our present calculations shows a good agreement in terms of the binding energies and the peak intensities in the both outer- and inner-valence regions.

\section{ACKNOWLEDGMENTS}

We are grateful to Dr. J. Meller and Mr. K. Toyota for their helpful discussions on this subject. This research was supported by a Grant-in-Aid for Scientific Research from the Ministry of Education, Science, Culture, and Sports.

${ }^{1}$ G. Scheibe and H. Grieneisen, Z. Phys. Chem. Abt. B 25, 52 (1934).

${ }^{2}$ L. W. Pickett, E. Paddock, and E. Sackter, J. Am. Chem. Soc. 63, 1073 (1941)

${ }^{3}$ W. C. Price and A. D. Walsh, Proc. R. Soc. London, Ser. A 179, 201 (1941).

${ }^{4}$ R. P. Frueholz, W. M. Flicker, O. A. Mosher, and A. Kuppermann, J. Chem. Phys. 70, 2003 (1979).

${ }^{5}$ P. J. Derrick, L. Asbrink, O. Edqvist, B.-O. Jonsson, and E. Lindholm, Int. J. Mass Spectrom. Ion Phys. 6, 203 (1971).

${ }^{6}$ H. Nakatsuji, O. Kitao, and T. Yonezawa, J. Chem. Phys. 83, 723 (1985).

${ }^{7}$ V. Galasso, Chem. Phys. 153, 13 (1991).

${ }^{8}$ L. Serrano-Andres, M. Merchan, I. Nebot-Gil, B. O. Roos, and M. Fulscher, J. Am. Chem. Soc. 115, 6184 (1993)

${ }^{9}$ H. Nakano, T. Tsuneda, T. Hashimoto, and K. Hirao, J. Chem. Phys. 104, 2312 (1996).

${ }^{10}$ H. Nakatsuji, Chem. Phys. Lett. 177, 331 (1991).

${ }^{11}$ H. Nakatsuji and K. Hirao, J. Chem. Phys. 68, 2053 (1978).

${ }^{12}$ H. Nakatsuji, Chem. Phys. Lett. 59, 362 (1978).

${ }^{13}$ H. Nakatsuji, Chem. Phys. Lett. 67, 329 (1979).

${ }^{14} \mathrm{H}$. Nakatsuji, in Computational Chemistry: Reviews of Current Trends, edited by J. Leszczynski (World Scientific, Singapore, 1997), Vol. 2.

${ }^{15}$ K. Hirao and H. Nakatsuji, Chem. Phys. Lett. 79, 292 (1981).

${ }^{16}$ H. Nakatsuji, Chem. Phys. 75, 425 (1983).

${ }^{17}$ H. Nakatsuji, J. Chem. Phys. 83, 5743 (1985).

${ }^{18}$ H. Nakatsuji, M. Hada, M. Ehara, J. Hasegawa, T. Nakajima, H. Nakai, O. Kitao, and K. Toyota, SAC/SAC-CI program system (SAC-CI96) for calculating ground, excited, ionized, and electron attached states and singlet to septet spin multiplicities.

${ }^{19}$ LANDOLT-BORNSTEIN, Numerical Data and Functional Relationships in Science and Technology, New Series, Group II: Atomic and Molecular Physics, edited by J. H. Callomon, E. Hirota, K. Kuchitsu, W. J. Lafferty, A. G. Maki, and C. S. Pote (Springer-Verlag, Berlin-Heidelberg, 1976), Vol. 7.

${ }^{20}$ R. A. Kendall, T. H. Dunning, Jr., and R. J. Harrison, J. Chem. Phys. 96, 6796 (1992)

${ }^{21}$ T. H. Dunning Jr., J. Chem. Phys. 90, 1007 (1989).

${ }^{22}$ K. Kaufmann, W. Baumeister, and M. Jungen, J. Phys. B 22, 2223 (1989).

${ }^{23}$ A. B. Trofimov and J. Schirmer, Chem. Phys. 214, 153 (1997).

${ }^{24}$ Gaussian 98, Revision A.1, M. J. Frisch, G. W. Trucks, H. B. Schlegel et al., Gaussian, Inc., Pittsburgh, Pennsylvania, 1998.

${ }^{25}$ R. S. Mulliken, J. Chem. Phys. 7, 121 (1939).

${ }^{26}$ M. B. Robin, Higher Excited States of Polyatomic Molecules (Acadamic, New York, 1975), Vol. II, pp. 166-176.

${ }^{27}$ R. McDiarmid and A. Sabljic, J. Chem. Phys. 83, 2147 (1985).

${ }^{28} \mathrm{H}$. Nakatsuji, J. Wan, and M. Hada (unpublished).

${ }^{29}$ A. Sabljic, R. McDiarmid, and A. Gedanken, J. Phys. Chem. 96, 2442 (1992).

${ }^{30}$ D. F. Evans, J. Chem. Soc., 1735 (1960). 